\title{
ACHADOS ULTRA-SONOGRÁFICOS DA HEMORRAGIA INTRACRANIANA EM RECÉM-NASCIDOS PREMATUROS
}

\author{
Luciano Farage ${ }^{1}$, Marcelo Cardoso de Assis ${ }^{2}$
}

\begin{abstract}
RESUMO - A hemorragia intracraniana (HIC) é a manifestação mais comum no sistema nervoso central de recém-nascidos (RN) prematuros, especialmente os de peso menor que $1500 \mathrm{~g}$, ou com idade gestacional (IG) menor que 32 semanas. O local mais acometido é a matriz germinal e é classificado em graus por Papile et al. Foram analisados prospectivamente 50 RN pré-termo (IG <37 semanas) com diagnóstico de HIC ao exame ultra-sonográfico (US) transfontanelar. Eles foram classificados quanto à idade, sexo, idade gestacional, peso ao nascer, gravidade e evolução ultra-sonográfica da lesão. As crianças foram divididas em dois grupos (A: IG $\leq 33$ semanas e B: $34 \geq$ IG $<37$ semanas). No grupo A tivemos 34 RN ( 25 meninos) com IG média de 31 semanas e peso médio de $1308 \mathrm{~g}$. No grupo B tivemos 16 RN (2 meninos) com IG média de 34 semanas e peso médio de $1951 \mathrm{~g}$. A distribuição da HIC nos grupos foi: Grupo A - Grau I- 14, II-14, III-4 e IV- 2 e Gru po B - I- 12, II- 3, III-1. Não houve diferença estatística do grau da HIC entre meninos e meninas ou entre os g rupos de RN. As complicações foram mais comuns no grupo A, com um total de 12, contra 4 no Grupo B. 0 US se mostrou método eficiente no diagnóstico e acompanhamento dos RN com HIC.
\end{abstract}

PALAVRAS-CHAVE: ultra-som transfontanelar, hemorragia intracraniana, recém-nascido prematuro.

\section{Ultrasonic findings of intracranial hemorrhage in preterm neonates}

ABSTRACT - Intracranial hemorrhage ICH is one of the most common neurological events in pre - te rmnewborn ICH is associated with low birth weight $(<1500 \mathrm{~g})$ and gestational age (GA) at delivery ( $<32$ weeks). The most common site affected is the germinal matrix. Papile et al. classifies it at four grades. We analyzed, prospectively, 50 newborns (27 boys) with ultrasound diagnostic of ICH; all of them were pre-term (GA < 37 weeks). They were classified according to sex, gestational age, birth weight and degree of ICH. The child ren were divided into two groups: $A$ - $G A \leq 33$ weeks and B - 34-37 weeks. In group A there were 34 child ren ( 25 boys) wither mean GA of 31 weeks and birth weights average of $1308 \mathrm{~g}$. In group B there were 16 children ( 2 boys), mean GA 34 weeks and birth weight average of $1951 \mathrm{~g}$. The grades of ICH were: Group A I-14, II-14, III-4 and IV-2; Group B - I-12, II-3 and III-1. The complications were more common in group A with 12 than group B with 4 children. The lesions happen in greatest number and most severity in children with low birth weight and younger (low gestational age). Ultrasound has shown to be effective for diagnostic and follow up of those children.

KEY WORDS: ultrasound, intracranial hemorrhage, newborn.

A hemorragia intracraniana (HIC) é a enfermidade de maior prevalência do sistema nervoso central do recém-nascido pre matu ro(RNP). Diversos estudos demonstram que a idade gestacional ao nascimento, inferior a 32 semanas, e o baixo peso ao nascimento, inferior a $1500 \mathrm{~g}$, são os maiores $\mathrm{fa}$ to res de risco ${ }^{1-9}$. O local mais freqüentemente acometido, em prematuros, é a matriz germinal e sua classificação é dada pela escala de Papile et al. ${ }^{3}$. Vários estudos têm sido realizados no intuito de se avaliar o melhor método de imagem para diagnóstico e acompanhamento das HIC $4,5,8,10,11$.
A ultra-sonografia (US) transfontanelar apresenta-se como um destes métodos e possui como vantagens o seu baixo custo econômico, a versatilidade e mobilidade do aparelho que pode deslocar-se fisicamente tornando factível o exame à beira do leito e sua inocuidade, uma vez que neste método não existe emissão de radiação. Pode ser realizado repetidas vezes, sendo ainda desnecessária a sedação do paciente ${ }^{6,8}$. A desvantagem do método estaria na sua menor sensibilidade e especificidade para lesões parenquimatosas, especialmente alterações isquêmicas, quando comparado com a

Setor de Radiologia, Hospital de Clínicas, Faculdade de Medicina (Famed), Universidade Federal de Uberlândia, Uberlândia MG, Brasil (UFU): 'Ex-Médico-Residente de Radiologia e Diagnóstico por Imagem; ²Professor Adjunto de Neurologia, Departamento de Cirurgia, Famed, UFU. 
Tabela 1. Gênero, idade gestacional e peso ao nascimento dos recém-nascidos prétermo dos grupos $A$ e $B$.

\begin{tabular}{lcc}
\hline & \multicolumn{1}{c}{ Grupo A } & Grupo B \\
\hline Gênero & 25 masculino, 9 feminino & 2 masculino, 14 feminino \\
Idade gestacional & 31 sem $(26-33$ semanas $)$ & 34 sem $(34-36$ semanas $)$ \\
Peso de nascimento & $1308 \mathrm{~g}(580-2510 \mathrm{~g})$ & $1951 \mathrm{~g}(1320-2620 \mathrm{~g})$ \\
\hline
\end{tabular}

Tabela 2. Distribuição dos pacientes por grau das hemorragias intracranianas (HIC) nos grupos $A$ e $B$.

\begin{tabular}{lcc}
\hline Grau da HIC & Grupo A & Grupo B \\
\hline I & 14 & 12 \\
II & 14 & 3 \\
III & 4 & 1 \\
IV & 2 & \\
Total & 34 & 16 \\
\hline
\end{tabular}

Tabela 3. Distribuição das complicações pelos grupos $A$ e $B$.

\begin{tabular}{lcc}
\hline Complicações & Grupo A & Grupo B \\
\hline Hidrocefalia & 6 & 2 \\
Encefalomalácia & 5 & 2 \\
Óbito & 1 & - \\
Total & 12 & 4 \\
\hline
\end{tabular}

tomografia computadorizada (TC) e a ressonância magnética (RM) $)^{5,8,10,11}$. No entanto, esses dois métodos são mais caros, necessitam de deslocamento ao departamento de radiologia e exigem sedação da criança na maioria dos exames $4,5,8,10,11$. Em revisão da Academia Americana de Neurologia e da Sociedade de Neurologia Infantil, o US apresenta nível de evidência $B$ (provavelmente útil em populações selecionadas) em RNP com idade $<30$ semanas $^{8}$. As grandes vantagens da RM e TC na avaliação dos recém-nascidos com alterações hemorrágicas e isquêmicas são maior sensibilidade e especificidade e principalmente menor variabilidade inter-observador (índice kappa) ${ }^{10}$. Alguns estudos têm mostrado que a utilização, principalmente da RM, têm permitido diagnósticos de alterações encefálicas (isquemias e hemorragias) intra-útero, o que têm pe rmitido alguns questionamentos quanto à etiologia e o prognóstico das lesões ${ }^{8-10}$.

O presente estudo tem como objetivos estudar: a) demonstrar o aspecto da hemorragia intracraniana à US em RNP, classificando-as em graus segundo proposto por Papile et al ${ }^{1,3}$; b) comparar os achados da US craniana neste grupo de RNP dividindo-os segundo a idade gestacional; RNP acompanhar a evolução ultra-sonográfica dos RNP com hemorragia já diagnosticada.

\section{MÉTODO}

Foram analisados, prospectivamente, 50 RNP admi- tidos na Unidade Neonatal do Hospital de Clínicas da Universidade Federal de Uberlândia, no período comp reendido entre agosto de 2003 e março de 2004. Estes recém-nascidos foram submetidos ao exame de US transfontanelar no Setor de US e receberam o diagnóstico de HIC.

O critério para inclusão foi à presença da HIC ao exame ultra-sonográfico inicial (por indicação neonatal e sem a participação dos examinadores) e idade gestacional informada no prontuário menor do que 37 semanas. Foram excluídas as crianças que não realizaram exame de controle, seja ambulatorial (até seis meses após o exame inicial) ou de seguimento na mesma intemação (intervalo mínimo de 30 dias após o primeiro exame).

As informações clínicas utilizadas foram exclusivamente de dados antropométricos, como gênero, idade, peso ao nascer e ultra-sonográficos, como localização e grau da hemorragia.

A HIC foi classificada pela escala de Papile et al. em graus: no grau I (mais leve) ocorre hemorragia limitada à matriz germinal; no grau II há extravasamento para o sistema ventricula; no grau III já existe aumento do sistema ventricular; no grau IV há hemorragia no parênquima encefálico ${ }^{3}$.

Foram consideradas complicações os achados tanto de exames de seguimento imediato (na mesma internação) quanto tardio (em retorno ambulatorial).

Os grupos foram comparados através do teste do $\chi 2$ e admitido a nível de significância com valor de $p=0,05$.

O presente estudo foi aprovado pelo Comitê de Ética em Pesquisa da Universidade Federal de Uberlândia.

Técnica de exame - Todos os exames foram realizados pelo mesmo examinador, em aparelho Sonolite VersaPro, Siemens Medical, com transdutor convexo de 6,5 $\mathrm{MHz}$ e linear de 7,5 MHz nos planos coronal e sagital, através da fontanela anterior ${ }^{6}$.

\section{RESULTADOS}

Foram observados 50 recém-nascidos com HIC diagnosticada pela US. Para fins de analise, estes foram divididos em dois grupos ( $\mathrm{A}$ - pré-termo, $\mathrm{IG} \leq 33$ semanas e $B$ - quase-termo $34 \geq$ IG $<37$ semanas).

Na Tabela 1 encontram-se os dados referentes ao sexo, idade gestacional e peso ao nascimento. $\mathrm{Na}$ Tabela 2 tem-se a distribuição das hemorragias intracranianas por graus, segundo a classificação proposta por Papile et al. ${ }^{1,3}$. Na Tabela 3 encontram-se relatadas as complicações advindas da 
hemorragia intracraniana e que foram diagnosticadas nos exames evolutivos (todas as crianças realizaram exame evolutivo, mas apenas os resultados anormais são demonstrados nesta tabela).

\section{DISCUSSÃO}

A HIC em recém-nascidos prematuros é importante causa de mortalidade e morbidade, de incidência elevada neste grupo de pacientes. Freqüentemente associa-se à baixa idade gestacional e ao baixo peso ao nascimento ${ }^{1-9}$.

Em nosso estudo, quando os dois grupos de RNP foram comparados, observamos que no grupo $A$ (idade gestacional $\leq 33$ semanas) houve predomínio de RNP do gênero masculino, enquanto que no grupo $B$ (idade gestacional $34 \geq$ IG $<37$ semanas) houve predomínio de meninas. O predomínio de cada gênero em um dos grupos foi um achado eventual já que em outras séries publicadas este dado não é corroborado 2,4,5,11.

Quanto ao grau da hemorragia, considerando os pacientes do Grupo A, observamos maior número de casos nos graus I e II (14 pacientes - $42 \%$ - em cada Grau). No Grupo B houve predomínio absoluto do Grau I ( 12 casos - 75\% do total). Em rel ação aos graus da hemorragia, observamos que as hemorragias graus III e IV foram encontradas em $14 \%$ dos RNP estudados ${ }^{5}$, com predomínio nos pacientes pertencentes ao Grupo A (6 casos). Não houve diferença estatisticamente significativa entre os grupos em número e grau das $\mathrm{HIC}(\mathrm{p}=0,147)$, bem como não houve diferença entre os gêneros $(p=0,605)$.

Quanto às complicações (hidrocefalia e encefalomalácia), são mais freqüentes quanto mais elevado for o grau da hemorragia, segundo a literatura consultada ${ }^{2,6,8}$. Em nossa casuística isto se confirmou, pois dos oito pacientes que desenvolveram hidrocefalia pós-hemorragia seis eram do Grupo A e 2 dois do Grupo B e pertenciam ao grupo de hemorragias grau III e IV, à exceção de um paciente com Grau II (Grupo B). Dos sete pacientes de nossa casuística que apresentaram encefalomalácia (cinco no grupo $A$ e dois no Grupo B), todos eram provenientes dos graus II a IV.

Alguns trabalhos têm sido realizados para avaliar os efeitos cognitivos destas alterações encefálicas. Ohlweiler et al. ${ }^{12}$, analisando grupos de crianças pré-termo com HIC ou convulsão e comparando-as a crianças sem alterações neurológicas, encontraram significativo atraso do desenvolvimento neuropsicomotor no primeiro grupo ${ }^{13}$.

Durante todo o período do estudo e seguimen- to dos pacientes ocorreu um óbito em um RNP com hemorragia grau III por provável associação de complicações pulmonares e ressangramento cerebral.

O US como método de imagem tem algumas limitações, principalmente no grau II da HIC, que a p resenta menor acurácia que nos graus I, III e IV. Para alterações parenquimatosas císticas, o método a p resenta acurária de $100 \%$. Na revisão feita pela Academia Americana de Neurologia e pela Sociedade de Neurologia Pediátrica, o rastreamento com US em RNP é considerado como adequado para a população selecionada (recomendação nível B) ${ }^{8}$. A RM é mais sensível que o US. Em estudos nos quais a RM e o US foram realizados no mesmo dia, houve maior detecção de lesões na substância branca e hemorragias. Entretanto ainda não há dados suficientes que mostrem evolução diferente dos aspectos neuropsicológicos, sendo o grau de recomendação C (mais estudos são necessários). Não há ainda indicação de RM como rotina em exames de US alterados ${ }^{8}$.

Em conclusão, o ultra-som transfontanelar mostrou-se um método bastante eficiente para avaliação das hemorragias intracranianas e suas complicações ventriculares e periventriculares nos nossos casos.

\section{REFERÊNCIAS}

1. Assis MC, Machado HR. Ecografia transfontanelar com fluxo a cores em recém-nascidos prematuros. Arq Neuropsiquiatr 2004;62:68-74.

2. Hansen A, Leviton A. Labor and delivery characteristics and risks of cranial ultrasonographic abnormalities among very-low-birth-weight infants. The Developmental Epidemiology Network Investigators. Am J Obstet Gynecol 1999;181:997-1006.

3. Papile LA, Burstein J, Burstein R, Koffler H. Incidence and evolution of subependymal and intraventricular hemorrhage: a study of infants with birth weights less than 1,500 gm. J Pediatr 1978;92:529-534.

4. Roelants-van Rijn AM, Groenendaal F, Beek FJ, Eken P, van Haastert IC, de Vries LS. Parenchymal brain injury in the preterm infant: comparison of cranial ultrasound, MRI and neurodevelopmental outcome. Neuropediatrics 2001;32:80-89.

5. RutherfordMA, Pennock JM, Dubowitz LM. Cranial ultrasound and magnetic resonance imaging in hypoxic-ischaemic encephalopathy: a comparison with outcome. Dev Med Child Neurol 1994;36:813-825.

6. Seigel MJ. Ultra-sonografia: Pediátrica. Rio de Janeiro, Guanabara-Koogan, 2003.

7. Ve rgani P, Patane L, Doria P, et al. Risk factors for neonatal intraventricular haemorrhage in spontaneous prematurity at 32 weeks gestation or less. Placenta 2000;21:402-407.

8. Ment LR, Bada HS, Barnes P, et al. Practice parameter: neuroimaging of the neonate. Neurology 2002;58:1726-1738.

9. Ferreiro DM Neonatal brain injury. N Engl J Med 2004;1985-1995.

10. BlankenbergFG, Loh NN, Bracci P, et al. Sonography, CT, and MR imaging: a prospective comparison of neonates with suspected intracranial ischemia and hemorrhage. AJNR 2000;21:213-218.

11. Maalouf EF, Duggan PJ, Counsell SJ, et al. Comparison of findings on cranial ultrasound and magnetic resonance imaging in preterm infants. Pediatrics 2001;107:719-727.

12. Ohlweiler L, Silva AR, Barros SV, Riesgo R, Rotta NT. Influence of intracranial hemorrhage and neonatal seizures on the neurological and psychomotor development of prematureinfants at Hospital de Clínicas de Porto Alegre, Brazil. Arq Neuropsiquiatr 2003;61:902-905.

13. Sanchez-Stopiglia M, Moura-Ribeiro MVL, Marba S. Avaliação neurológica de recém-nascidos com hemorragia intraventricular e periventricular. Arq Neuropsiquiatr 1999;57:366-370. 